\title{
Demonstration of a Variable Optical Delay for a Recirculating Buffer by Using All-Optical Signal Processing
}

\author{
Y. Liu, M. T. Hill, R. Geldenhuys, N. Calabretta, H. de Waardt, G.-D. Khoe, Fellow, IEEE, and H. J. S. Dorren
}

\begin{abstract}
We demonstrate a variable optical delay by using a recirculating loop that is controlled by all-optical signal processing technology. This concept can be utilized in all-optical recirculating buffers. The variable optical delay is realized by using an optical threshold function and a wavelength converter. We show that in case of packet contention, one optical packet is recirculated four times in the loop and when the contention disappears, the packet is routed out of the optical loop.
\end{abstract}

Index Terms-Buffer memories, optical fiber communication, optical fiber delay lines, packet switching.

\section{INTRODUCTION}

O PTICAL buffering technology should be used to avoid optical packet contention in all-optical switched networks [1]. In these buffering configurations, the data packets are stored in fiber delay lines. In general, two types of buffers are used: travelling buffers and recirculating buffers [2]. In travelling buffers, the delay time is determined by the length of the optical delay line. Such configurations are investigated in [3], where optical travelling buffering is realized by using electronically controlled wavelength routing switches, and in [4], where an all-optical travelling buffer concept is realized by using an optical threshold function (OTF) in combination with a wavelength routing switch. The concept in [4] is extended to handle multipacket contention [5]. Travelling buffers have a disadvantage since a large number of delay lines are required to avoid packet loss under a heavy traffic load. This makes travelling buffers bulky. In recirculating buffers, however, the delay time is determined by the length of the loop and the circulation number. Hence, a single delay line can be used to realize a large delay by increasing the number of circulations. An important advantage of recirculating buffers over travelling buffers is that the physical size of the buffer is reduced. In [6], a variable optical delay is demonstrated by using optical delay lines and wavelength converters. After each circulation, the optical signal is converted to a new wavelength. A disadvantage of the approach presented in [6] is that for each circulation, a wavelength converter is required. This makes the system complicated and expensive. In [7], a variable optical delay is realized by using an optical delay line and one wavelength shifter.

Manuscript received December 23, 2003; revised February 23, 2004. This work was supported by the Netherlands Organization for Scientific Research (NWO), the Technology Foundation STW, and by the Ministry of Economic Affairs through, respectively, the NRC Photonics Grant and the Innovational Research Incentives Scheme Program.

The authors are with the COBRA Research Institute, Eindhoven University of Technology, 5600 MB Eindhoven, The Netherlands (e-mail: Y.Liu @tue.nl).

Digital Object Identifier 10.1109/LPT.2004.829536

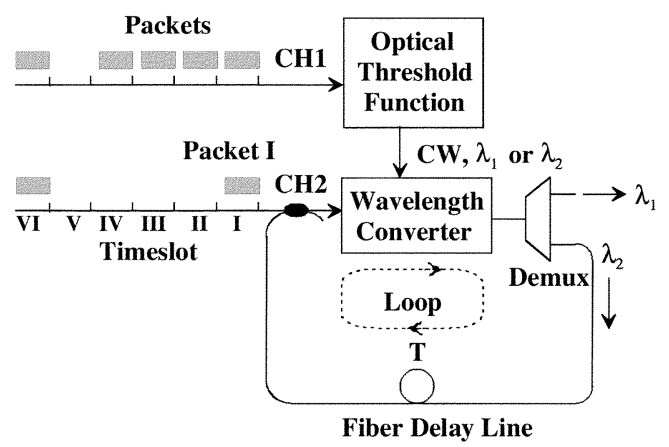

Fig. 1. Functional diagram of the variable optical delay concept. $T$ : the delay time equal to one timeslot. Demux: demultiplexer.

The number of circulations is determined by the initial wavelength of the optical input signal, the final output wavelength, and the wavelength shift in each circulation. The wavelength shifter is made out of two wavelength converters. This scheme requires a high-power pump light $(28.9 \mathrm{dBm})$ that is phase modulated [7]. In this letter, we demonstrate a variable optical delay by employing a recirculating loop that is controlled by all-optical signal processing technology. This concept can be utilized in recirculating buffers, as described in [2]. We show that an OTF (as presented in [4]) in combination with a single optical wavelength converter based on nonlinear polarization rotation in a semiconductor optical amplifier (SOA) can be used to realize a variable optical delay. The variable delay time is achieved by altering the number of circulations of the packet in the loop. In our approach, the circulation number is determined by the traffic situation. Thus, the delay time of the packet depends on the traffic, which makes the system flexible and suitable for recirculating buffers. We demonstrate that an optical packet is recirculated in an optical loop four times when packet contention occurs. When the contention disappears, the packet is routed out of the optical loop.

\section{OPERATING PRINCIPLE}

The variable optical delay concept is presented schematically in Fig. 1. We assume that the packets arrive in a synchronized fashion and also that the packets in Channel $2(\mathrm{CH} 2)$ have the lowest priority to pass the node. The packets in Channel 1 (CH1) have the highest priority to pass the node. Thus, if packet contention takes place, the packets in $\mathrm{CH} 2$ have to be sent into the loop. The packets in $\mathrm{CH} 2$ can only pass through the node if there is no packet in $\mathrm{CH} 1$ that causes packet contention in a specific timeslot. The packets in $\mathrm{CH} 1$ are injected into an OTF that acts as an optical arbiter to decide if packet contention occurs. 


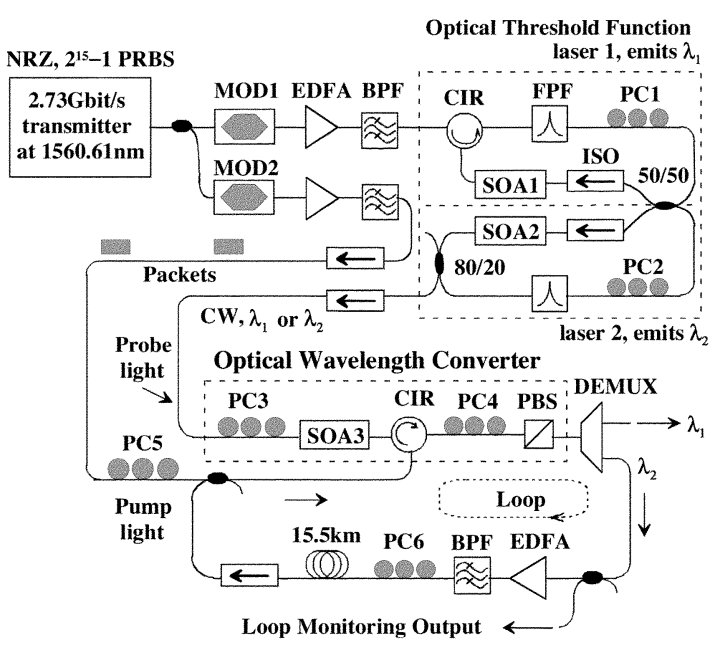

Fig. 2. Experimental setup. BPF: bandpass filter. FPF: Fabry-Pérot filter. PC: polarization controller. ISO: isolator. CIR: circulator. PBS: polarization beam splitter. Demux: demultiplexer.

The first essential building block of this concept is an OTF. The OTF is made out of two coupled lasers and is described in detail in [4] and [8]. The OTF is depicted in Fig. 2. It is shown in [4] and [8] that the OTF can have two possible states. In State 1, light from Laser 1 (lasing at wavelength $\lambda_{1}$ ) suppresses lasing in Laser 2 (lasing at wavelength $\lambda_{2}$ ). This means that the OTF outputs continuous-wave (CW) light at wavelength $\lambda_{1}$. Conversely, in State 2, light from Laser 2 suppresses lasing in Laser 1 , thus, the OTF outputs CW light at wavelength $\lambda_{2}$. The system is asymmetrical so that the OTF is in State 1 if no external light is injected. If external light is injected into Laser 1, lasing in Laser 1 is stopped and the OTF switches to State 2. The OTF remains in State 2 as long as external light is injected into Laser 1. If the injection of external light is stopped, the OTF switches back to State 1 [4], [8].

The second essential building block is a wavelength converter that is based on nonlinear polarization rotation in a single SOA, as described in detail in [9]. The configuration is shown in Fig. 2. It has been demonstrated in [9] that this wavelength converter can realize noninverted conversion with reshaping ability, and also can convert the signal into the same wavelength.

The system shown in Fig. 1 can function as a variable optical delay in a recirculating loop as follows. First, we assume that the synchronized data packets arrive at the node in distinct timeslots. The delay time introduced by the fiber delay line is exactly one timeslot. The $\mathrm{CW}$ light outputting from the OTF controls a wavelength converter that in turn converts the wavelength of packets that arrive in $\mathrm{CH} 2$. The optical demultiplexer is used to route packets into a specific port, depending on the wavelength of the packets. Suppose that in Timeslot I, packets in $\mathrm{CH} 1$ and $\mathrm{CH} 2$ are present, so that packet contention takes place. The packet in $\mathrm{CH} 1$ is injected in the OTF, thus, the OTF switches its state to output CW light at wavelength $\lambda_{2}$. Hence, the wavelength of Packet I is converted to $\lambda_{2}$ so that Packet I is routed into an optical loop which delays the packet for the duration of one timeslot. In Timeslot II, the OTF also outputs CW light at wavelength $\lambda_{2}$ due to the presence of a packet in $\mathrm{CH} 1$. Thus, Packet I, which was already converted to wavelength $\lambda_{2}$ and delayed by one timeslot, is reconverted to wavelength $\lambda_{2}$ and delayed for another timeslot in the delay loop. A similar situation takes place in Timeslot III and IV, but in Timeslot V, the

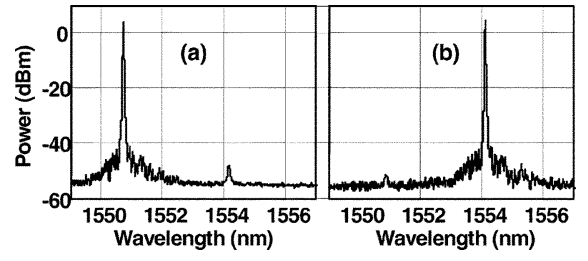

Fig. 3. Measured spectral output of the OTF without [Fig. 3(a)] and with [Fig. 3(b)] the presence of external light.

OTF outputs $\lambda_{1}$ due to the absence of a packet in CH1. Hence, Packet I is reconverted from wavelength $\lambda_{2}$ into $\lambda_{1}$ and is routed out of the loop.

It can be observed that the delay time of the packets in $\mathrm{CH} 2$ is determined by the traffic situation. This variable optical delay concept can be applied in [2], where a number of variable optical delay units are employed to construct a recirculating buffer.

\section{EXPERIMENTS}

The experimental setup is shown in Fig. 2. The bit rate of the data stream is $2.73 \mathrm{~Gb} / \mathrm{s}$, and the wavelength is 1560.61 $\mathrm{nm}$. The bit patterns in the data stream have a nonreturn-to-zero data format and form a $2^{15}-1$ pseudorandom binary sequence. The external modulators (MODs) are used to generate optical packets. The packets that are generated by MOD1 represent the packets in CH1 (shown in Fig. 1), and are injected into the OTF via a circulator. The packets that are generated by MOD2 represent the packets in $\mathrm{CH} 2$ (shown in Fig. 1), and are directed to an optical wavelength converter via a $3-\mathrm{dB}$ coupler and a circulator. The packets have a duration of $57.5 \mu \mathrm{s}$, and the guard time between each packet is $20 \mu \mathrm{s}$. The output of the OTF is fed into the optical wavelength converter. The injection current of SOA 3 in the wavelength converter is $300 \mathrm{~mA}$. The optical power of probe light that outputs from the OTF is $1 \mathrm{dBm}$ and the average optical power of a packet that is injected into SOA 3 is $1 \mathrm{dBm}$. The length of the optical loop is about $15.5 \mathrm{~km}$, which is equivalent with a delay of $77.5 \mu$ s for the packets. An erbium-doped fiber amplifier (EDFA) in the loop provides 19-dB gain for the packet.

The OTF is depicted in the upper dashed box of Fig. 2. The OTF consists of two coupled ring lasers, in which SOAs act as laser gain media. The wavelengths are selected by Fabry-Pérot filters. Optical isolators are used to ensure lasing in one direction. The lasers in the OTF are constructed from standard commercially available fiber pigtailed components, which makes that the total length of each laser cavity is over ten meters. The wavelength of each laser is $\lambda_{1}=1550.92 \mathrm{~nm}$ and $\lambda_{2}=1554.05 \mathrm{~nm}$. The SOA injection currents are set in such a way that the system is asymmetric. The SOA injection currents are $354 \mathrm{~mA}$ for SOA 1 (the threshold current of Laser 1 is $71 \mathrm{~mA}$ ) and $247 \mathrm{~mA}$ for SOA 2 (the threshold current of Laser 2 is $85 \mathrm{~mA}$ ), respectively. The spectral output of the OTF is presented in Fig. 3. It follows from Fig. 3(a) that Laser $1(1550.92 \mathrm{~nm})$ is dominant if no external light is injected. In Fig. 3(b), the spectrum of the OTF is shown if $10 \mathrm{dBm}$ of external light $(1560.61 \mathrm{~nm})$ is injected into Laser 1; it follows that Laser $2(1554.05 \mathrm{~nm})$ is dominant. However, the OTF returns to State 1 if the external injection is stopped. It can be observed from Fig. 3 that the contrast ratio between the two states in the OTF is over $50 \mathrm{~dB}$. 


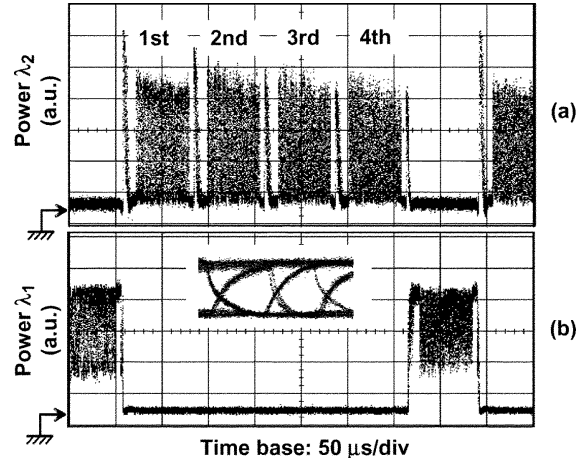

Fig. 4. (a) Oscilloscope trace showing that the packet circulates in the loop four times. (b) Oscilloscope trace showing that the packet is converted to $\lambda_{1}$ and routed out of the loop after recirculating in the loop four times. The inset picture is an eye diagram of the converted output data in Fig. 4(b).

We demonstrate a variable optical delay that has the same traffic situation as discussed in Section II. Therefore, optical packets are created in repeated five distinct timeslots. In Timeslots I-IV, the optical packets that are created in $\mathrm{CH} 1$ are injected into the OTF. As explained before, these packets cause the OTF to switch its state to State 2. Thus, in Timeslots I-IV, the OTF outputs CW light at wavelength $\lambda_{2}$. As a result, Packet I is converted in these timeslots to wavelength $\lambda_{2}$ and routed into the optical loop where it remains for four circulations. It is shown in Fig. 4(a) (via the loop monitoring output) that Packet I circulates in the loop for four timeslots (from Timeslot I to IV). In Timeslot $\mathrm{V}$, no packet is present in $\mathrm{CH} 1$ and, thus, the OTF switches back to output CW light at wavelength $\lambda_{1}$. Hence, Packet I is converted into $\lambda_{1}$ (inverted conversion) and passes through the node, as shown in Fig. 4(b). The inset picture shows the eye pattern of the packet after the packet has been switched out of the loop. A widely open eye is obtained. Fig. 4(a) shows a different wavelength conversion polarity compared to Fig. 4(b). This is because the conversion polarity of our wavelength conversion is related to the polarization of the $\mathrm{CW}$ light that outputs from the OTF. In the OTF, the polarization of the lasing light in each state is different due to the long laser cavities. The wavelength converter is optimized to realize noninverted conversion at wavelength $\lambda_{2}$ [see Fig. 4(a)] to fully benefit from the reshaping ability [9]. This is important to prevent the packet from signal degradation due to accumulated amplified spontaneous emission noise generated by the EDFA in the loop [6], [7]. When the OTF outputs light at wavelength $\lambda_{1}$, the polarization of the OTF output light is changed so that inverted conversion takes place [see Fig. 4(b)]. In addition, it takes several microseconds for the OTF to change state due to the long cavities. This leads to a $20-\mu$ s guard time between the packets. The slow switching time causes the large spikes in the guard time, as shown in Fig. 4(a). Photonic integration of the OTF could prevent both unwanted effects, since an integrated OTF can output purely transverse-electric polarized light in both states. As a result, wavelength conversion in Fig. 4(a) and (b) can both be noninverted. If no packet is sent into the wavelength converter, noninverted wavelength conversion ensures that no optical power will appear at the output of the loop. Also, integrated OTFs could attain speeds about a few gigahertz, so that the large spikes in Fig. 4(a) will be eliminated and the packet length could be reduced to within a hundred nanoseconds.
In the experiment, only four circulations have been demonstrated because the pulse pattern generator we used can only generate five preprogrammed data packets (since the packet is very long). The maximum circulation number is determined by the quality of the OTF, the reshaping ability of the wavelength converter, the noise figure of the EDFA, the packet length, and the polarization state of the light in the loop. We have observed that a packet with a length of $9 \mu$ s has been successfully circulated in the loop 15 times by using electronically controlled distributed feedback lasers instead of the OTF. We believe that at least several tens of circulations can be achieved by using integrated versions of the OTF and reducing the packet length to hundreds nanoseconds. The wavelength converter in our concept can be replaced by an integrated Mach-Zehnder interferometric wavelength converter, which has shown remarkable reshaping ability [6]. Thus, the maximum circulation time can be further increased.

A particular bit rate $(2.73 \mathrm{~Gb} / \mathrm{s})$ is used because the sum of the packet length and the guard time between each packet needs to be equal to one timeslot. In principle, the bit rate is only limited by the wavelength converter, and could reach more than $10 \mathrm{~Gb} / \mathrm{s}$ [9].

\section{CONCLUSION}

We have proposed a variable optical delay in a recirculating loop by using entirely all-optical signal processing technology. This concept can be utilized in all-optical recirculating buffers. We have shown that an OTF in combination with an optical wavelength converter can be used to realize a variable optical delay. The OTF showed a contrast ratio of more than $50 \mathrm{~dB}$ between the output states. An optical packet was circulated in an optical loop four times when packet contention occurred, and was routed out of the loop when the contention disappeared. A clear open eye after the recirculating loop was obtained.

\section{REFERENCES}

[1] D. K. Hunter, M. C. Chia, and I. Andonovic, "Buffering in optical packet switches," J. Lightwave Technol., vol. 16, pp. 2081-2094, Dec. 1998.

[2] W. D. Zhong and R. S. Tucker, "Wavelength routing-based photonic packet buffers and their applications in photonic packet switching systems," J. Lightwave Technol., vol. 16, pp. 1737-1745, Oct. 1998.

[3] C. Guillemot et al., "Transparent optical packet switching: The European ACTS KEOPS project approach," J. Lightwave Technol., vol. 16, pp. 2117-2134, Dec. 1998.

[4] Y. Liu, M. T. Hill, N. Calabretta, H. de Waardt, G. D. Khoe, and H. J. S. Dorren, "All-optical buffering in all-optical packet switched crossconnects," IEEE Photon. Technol. Lett., vol. 14, pp. 849-851, June 2002.

[5] Y. Liu, M. T. Hill, H. de Waardt, G. D. Khoe, and H. J. S. Dorren, "Alloptical buffering using laser neural networks," IEEE Photon. Technol. Lett., vol. 15, pp. 596-598, Apr. 2003.

[6] T. Sakamoto, K. Noguchi, R. Sato, A. Okada, Y. Sakai, and M. Matsuoka, "Variable optical delay circuit using wavelength converters," Electron. Lett., vol. 37, pp. 454-455, Mar. 2001.

[7] T. Sakamoto, A. Okada, O. Moriwaki, M. Matsuoka, and K. Kikuchi, "Variable optical delay circuit using highly nonlinear fiber parametric wavelength converters," Electron. Lett., vol. 39, pp. 198-200, Jan. 2003.

[8] M. T. Hill, E. E. E. Frietman, H. de Waardt, G. D. Khoe, and H. J. S. Dorren, "All fiber-optic neural network using coupled SOA based ring lasers," IEEE Trans. Neural Networks, vol. 13, pp. 1504-1513, Nov. 2002.

[9] Y. Liu, M. T. Hill, E. Tangdiongga, H. de Waardt, N. Calabretta, G. D. Khoe, and H. J. S. Dorren, "Wavelength conversion using nonlinear polarization rotation in a single semiconductor optical amplifier," IEEE Photon. Technol. Lett., vol. 15, pp. 90-92, Jan. 2003. 